\title{
Radar Waveform Design For High Resolution Doppler Target Detection
}

\author{
Mohd. Moazzam Moinuddin, \\ Professor of ECE \& Principal, Noor College of Engineering \& Technology, Shadnagar, Mahboobnagar (dt.), \\ A.P, India
}

\begin{abstract}
This paper addresses the problem of optimum waveform design for multiple target detection. The notion of poly-semantic radar, which gave improved performance through coincidence detection, is analyzed for high resolution radar system in presence of high density additive noise and Doppler shift. These sequences are optimized by employing Hamming backtrack algorithm (HBT). The detection capability of poly-semantic sequences is further improved through coincidence detection of the return signal. The simulation results show that the proposed sequences give improved robustness of noise and Doppler shift for HRR target detection compared to conventional pulse compression sequences.
\end{abstract}

Keywords: Hamming backtrack algorithm, high resolution radar, target detection.

\section{Introduction}

In high resolution radar (HRR) systems, there is a need to employ sequences of larger lengths to achieve high pulse compression ratios [1], [2]. The Optimal binary codes (OBC) including Barker code (B13) and Golay codes [3] provide significant advantages in terms of detection and sidelobe suppression [4], but these codes are available at lower lengths less than 60. Earlier the generation of optimal sequences at higher length up to 5000 is developed for poly-alphabetic and bi-alphabetic sequences [5]-[7]. In poly-alphabetic radar [5] what is transmitted is a specially designed binary sequence so that there is no change in the transmission technology. On reception, it is decoded before further processing. This is a change in the current practice, the resistance to which has weakened, as decoding before further processing is also required by neural network processing [8]-[10] of radar return signal for additional advantage. After decoding, the return signal is subjected to multiple interpretations. Here, a binary sequence is transmitted, but through poly-gram reading, it can also be interpreted as quaternary and octal sequences. Thus, it is as if one sequence is physically transmitted, but three sequences are notionally transmitted and received. They can therefore be processed separately at the receiver to set up coincidence detection. In poly-alphabetic sequence design a bigram viewed as a quaternary element or a trigram viewed as an octal element is some what of a constrained concept. The Quaternary and octal elements as independent entities would have 3 and 7 first order Hamming neighbors, but bigrams and trigrams on the substratum of binary monograms, which undergo Hamming scan have only two and three first order Hamming neighbors. Thus, the higher order poly-gram interpretations have a disadvantage in Hamming scan. Also, the enlarged alphabets deteriorate the noise and Doppler robustness at higher lengths in poly-alphabetic sequence [6].

In order to overcome these drawbacks and to restrict the enlarged alphabets of poly- alphabetic sequence to binary, the poly-semantic sequences are proposed [11]. These sequences are mono-alphabetic nature of poly-alphabetic sequences. The work presented in [11] did not discuss about noise rejection ability of these sequences for detection a target in noise environment conditions. The work presented in this paper is an attempt to evaluate the detection ability of mono-alphabetic poly-semantic sequences for the application to high resolution radar in presence of high dense noise and Doppler frequency. In this paper, the detection performance of the detected signal in presence of additive noise and Doppler shift is evaluated in terms of figure of merit.

The figure of merit is defined [12] as,

$$
F^{(m)}=\frac{\overline{C^{(m)}(0)}-\max _{k \neq 0}\left[\overline{C^{(m)}(k)}\right]}{\overline{C^{(m)}(0)}}
$$

Here ' $m$ ' represents the number of bit errors obtained in the sequence. Thus, the figure of merit in (1) is defined in the context, when known number decoding errors are present in the detected signal. It is assume that distortion due to propagation delay is ignored. Also, the additive noise is independent with transmitted signal. But, in real time situation, the received signal is corrupted by random noise with unknown noise 
strength. If the additive noise exceeds the threshold level (at the detector), the received sequence is not true replica of transmitted signal. The resulting signal at the output of the detector will undergo any number of bit errors. Then the optimal waveform design problem is solved by redefining the measure of performance in (1) by taking into the effect of additive random noise at given signal to noise ratio $(\eta)$ as discussed in Sec. II.

\section{Design Algorithm And Associated Concepts}

The concept of mono-alphabetic poly-semanticism [11] is similar to adaptation of the selfcooperative sequences. A binary sequence $\mathrm{S}$ with good autocorrelation properties is designed. It is doubled in length by interleaving another binary sequence $T_{1}$ whose elements are so chosen that enlarged sequence is good. Yet another sequence $T_{2}$ is interleaved to triple the length and the elements of former are so chosen that the new enlarged $\mathrm{T}_{3}$ sequence has good autocorrelation.

Let, $\mathrm{S}=\left[\mathrm{S}_{0}, \mathrm{~S}_{1}, \mathrm{~S}_{2}, \ldots, \mathrm{S}_{\mathrm{N}-1}\right]$

Be a transmitted signal of length $\mathrm{N}$,

$\mathrm{R}=\left[\mathrm{R}_{0}, \mathrm{R}_{1}, \mathrm{R}_{2}, \ldots, \mathrm{R}_{\mathrm{N}-1}\right]$

Is received signal. Here, $\mathrm{R}=\mathrm{S}+\mathrm{W}$; where $\mathrm{W}$ is the additive noise signal at given $\eta$.

Now, (1) is redefined as

$$
F_{\eta}=\frac{\overline{C_{\eta}(0)}-\max _{k \neq 0}\left[\overline{C_{\eta}(k)}\right]}{\overline{C_{\eta}(0)}}
$$

Where, $F_{\eta}$ is the figures of merit at given $\eta$.

The cross correlation between $S \& R$ at given $\eta$ is

$$
\begin{aligned}
& c_{\eta}(k)=\sum_{i=0}^{N-1-k} s_{i} r_{i+k}, k=0,1,2, \ldots, N-1 \\
& \text { Also, } F_{\eta}=1-\frac{\max _{k \neq 0}\left[\overline{C_{\eta}(k)}\right]}{\overline{C_{\eta}(0)}} \text { or } \quad F_{\eta}=1-\frac{1}{D_{\eta}} \\
& \text { Where, } D_{\eta}=\frac{\overline{C_{\eta}(0)}}{\max _{k \neq 0}\left[\overline{C_{\eta}(k)}\right]}
\end{aligned}
$$

Is the discrimination at given $\eta$.

The over head bars in (4) \& (6) denote the averaging over the ensemble of R. The work presented in this paper considers the ensemble of $\mathrm{R}$ with 100 runs of additive noise signals in order to obtain more accurate performance. Here, $F_{\eta}$ is a monotone function of $D_{\eta}$ as in (6). When $D_{\eta}$ goes to infinity, $F_{\eta}$ becomes unity.

The range of $F_{\eta}$ is from 0 to 1 , making $F_{\eta}$ a non-euphuistic measure.

In the detection process by employing coincidence detection, the return signal $\mathrm{R}$ is triply processed to exploit the goodness at three different stages of construction. The criterion of goodness, which is used for design, takes into account the interaction of the three interleaved sequences $T_{1}, T_{2}$, and $T_{3}$.

\section{Hamming Backtrack Algorithm For PSS}

To optimize the performance of goodness, the poly-semantic sequences should undergo Hamming scan [5] by considering figure of merit as desideratum. The Hamming scan algorithm may not perform recursive search among all these Hamming neighbors and results in suboptimal solution to the signal design problem. When poly-semantic Hamming scan yields no sequence with a figure of merit better than the previous sequence, the backtracking Hamming scan algorithm can be employed to improve further the objective function of the resulting sequence. It considers a prescribed number $\mathrm{n}$ called span of the best Hamming neighbors (though they are all inferior to starting sequence) and improves them separately by repeated recursive Hamming scan, say c times (called climb). If some sequences superior to the starting poly-semantic sequence results, the best among them is selected. A span of 6 and a climb of 2 is used in the proposed 
algorithm. If the Hamming backtracking succeeds in improving the value of figure of merit, the search can resume by further application of poly-semantic Hamming scan.

\section{Phase Reversal Effect Due To Doppler Shift}

Another advantage of poly-semantic sequence arises because of its bi-phase mono-alphabetic nature. The bit error due to Doppler frequency occurs when the phase shift of the pulse exceeds $\pm \pi / 2$ unlike the poly phase sequence which results into a bit error when phase shift exceeds $\pm \pi / \mathrm{M}$, where $\mathrm{M}$ indicates the number of phase levels in the sequence. Fig. 1(a) shows the range of phase shift without bit errors for bi-phase sequences $(\mathrm{M}=2)$ and Fig. 1(b) for poly-phase sequences with $\mathrm{M}=4$.

In noise free environments, the phase shift added due to Doppler to the sub-pulses is monotonic function as required for goodness of measure. In poly-semantic sequences of length $\mathrm{N}$, the maximum phase shift allowed on each sub-pulse of duration $\tau=T / \mathrm{N}$ sec without bit errors is less than $\pm \pi / 2 \mathrm{~N}$. Where as in poly-phase sequences the maximum allowable phase shift is less than $\pm \pi / \mathrm{MN}$ without bit errors. Therefore the polysemantic sequences have M/2 times more Doppler tolerance when compared to poly- phase sequences.

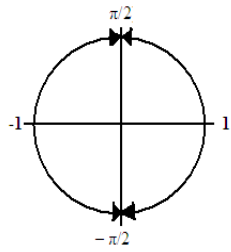

(a)

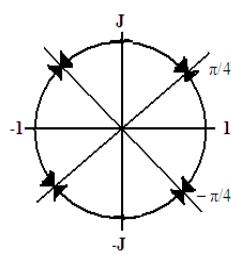

(b)

Fig. 1 Range Of Phase Shift without Bit Errors (A) For Bi-Phase Sequences (M=2) (B) Poly-Phase Sequences With $M=4$.

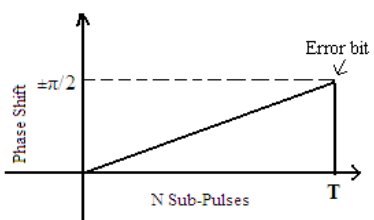

(a)

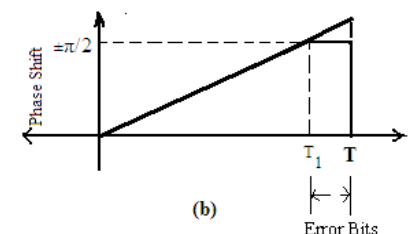

(b)

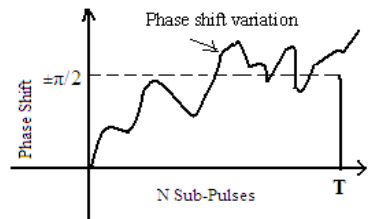

(c)

Fig. 2 Doppler Phase Shift On Received Bi-Phase Sequences (A) When Phase Shift Is Equal To $\pm \Pi / 2$ (B) When Phase Shift Is Greater Than $\pm \Pi / 2$ (C) When Additive Noise Is Added Along With Doppler Phase Shift.

In poly-semantic sequences, when the phase shift is equal to $\pm \pi / 2$, the last sub-pulse in the sequence takes phase reversal. So the last bit produces an error as shown in Fig. 2 (a). When the phase shift exceeds $\pm \pi / 2$, the bits within the period $T_{l} \& T$ (where, $T_{l}<T$ ) in Fig. 2(b) results into an error.

\section{Poly-Semantic Radar Signal Processor}

The generation of poly-semantic sequences is completed in two steps: first one using restricted (selective) Hamming backtracking scan for interspersed binary sequences and the second, using a complete Hamming backtracking scan with an appropriate joint objective function, which takes into the account of correlation properties between the received sequence $\mathrm{R}$ and predefined interleaved sequences in the process of signal design. The block schematic diagram of poly-semantic radar signal processor at the transmitter is shown in Fig. 3 


\section{First Step In The Signal Design}

Consider, optimal binary codes or randomly generated binary codes of length $\mathrm{N}$, given by

$$
\text { And } \quad \begin{aligned}
\mathrm{S}_{1}=\mathrm{A} & =\left[\mathrm{a}_{\mathrm{j}}\right] \\
\mathrm{B} & =\left[\mathrm{b}_{\mathrm{j}}\right] \\
\mathrm{C} & =\left[\mathrm{c}_{\mathrm{j}}\right]
\end{aligned}
$$

Where, $\mathrm{j}=0,1,2,3, \ldots, \mathrm{N}-1$.

The elements of these sequences are drawn from alphabet $\{-1,+1\}$.

The sequence $\mathrm{S}_{1}$ is mutated viz. $+\rightarrow-,-\rightarrow+$ using Hamming backtracking scan algorithm to get optimum figure of merit. The sequences $S_{2}$ of length $2 \mathrm{~N}$ and $S_{3}$ of length $3 \mathrm{~N}$ are generated by interleaving the elements of $S_{1} \& B$ and $S_{2} \& C$ respectively as shown in Fig. 3(a). Therefore

$$
\mathrm{S}_{2}=\left[\mathrm{a}_{\mathrm{j}} \mathrm{b}_{\mathrm{j}}\right]
$$

And $\mathrm{S}_{3}=\left[\mathrm{a}_{\mathrm{j}} \mathrm{b}_{\mathrm{j}} \mathrm{c}_{\mathrm{j}}\right]$

Where, $\mathrm{j}=0,1,2,3, \ldots, \mathrm{N}-1$.

A selective Hamming backtracking scan algorithm [10] is applied on the sequences $S_{2}$ and $S_{3}$, so that the figure of merit of the output sequence is optimized. This algorithm performs mutations only on the embedded elements, i.e., $b_{0}, b_{1}, b_{2}, b_{3} \ldots$ of the sequence $S_{2}$, and $c_{0}, c_{1}, c_{2}, c_{3} \ldots$ of the sequence $S_{3}$, without disturbing the other elements.

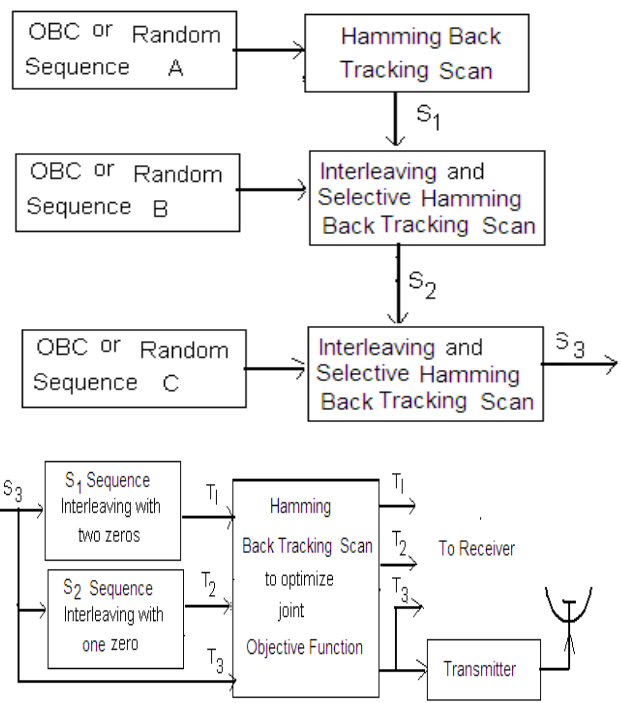

(a)

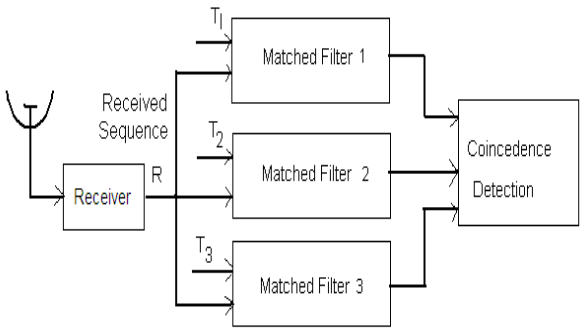

(b)

Fig. 3 Block Schematic Diagram of Poly-Semantic Radar Signal Processor (A) Transmitter Receiver.

\section{Second Step In The Signal Design}

The sequence $S_{3}$ is interspersed by binary sequences $S_{1}$ and $S_{2}$. It is equivalent to three sequences with good autocorrelation properties being transmitted in the form of $S_{3}$. On reception, the received waveform is decoded into binary sequence $(\mathrm{R})$ and the cross correlation is computed in discrete mode. The decoded sequence $\mathrm{R}$ is cross correlated in the receiver with three pre designed sequences, given by

$\mathrm{T}_{1}=\left[\mathrm{a}_{0}, 0,0, \mathrm{a}_{1}, 0,0, \mathrm{a}_{2}, 0,0 \ldots \mathrm{a}_{\mathrm{n}-1}, 0,0\right]$

$\mathrm{T}_{2}=\left[\mathrm{a}_{0}, \mathrm{~b}_{0}, 0, \mathrm{a}_{1}, \mathrm{~b}_{1}, 0, \mathrm{a}_{2}, \mathrm{~b}_{2}, 0 \ldots \mathrm{a}_{\mathrm{n}-1,1}, \mathrm{~b}_{\mathrm{n}-1,1}, 0\right]$

$\mathrm{T}_{3}=\left[\mathrm{a}_{0}, \mathrm{~b}_{0}, \mathrm{c}_{0}, \mathrm{a}_{1}, \mathrm{~b}_{1}, \mathrm{c}_{1}, \mathrm{a}_{2}, \ldots \mathrm{a}_{\mathrm{n}-1}, \mathrm{~b}_{\mathrm{n}-1}, \mathrm{c}_{\mathrm{n}-1}\right]$ 
The Hamming scan algorithm is applied on $\mathrm{T}_{1}, \mathrm{~T}_{2}$, and $\mathrm{T}_{3}$ for optimizing the joint figure of merit $\mathrm{F}=\left(\mathrm{F}_{1}+\mathrm{F}_{2}+\mathrm{F}_{3}\right) / 3$. Here, $\mathrm{F}_{1}, \mathrm{~F}_{2}$, and $\mathrm{F}_{3}$ are obtained by cross correlation of the sequences $\mathrm{S}_{3} \& \mathrm{~T}_{1}, \mathrm{~S}_{3} \& \mathrm{~T}_{2}$, and $S_{3} \& T_{3}$ respectively. The good figure of merit properties of these three interpretations are jointly used through coincidence detection for the detection of target. The binary sequence $S_{3}\left(T_{3}\right)$ is transmitted as a waveform.

The signal processing system at the receiver is shown in Fig. 3(b). On reception, the received waveform which is perturbed by Gaussian noise and / or Doppler shift is decoded into binary sequence (R). The received binary sequence $R$ is cross correlated with three embedded sequences $T_{1}, T_{2}$, and $T_{3}\left(\right.$ or $S_{3}$ ) in three channels separately. The three cross correlation peaks in three channels coincide, which simultaneously indicates the presence of the target (Fig. 9). It can also be observed that the time sidelobes in three channels do not align. This in turn reduces the degree of false alarm because of time sidelobes in the return signal.

\section{Noise Robustness}

\section{Simulation Results and Performance Evaluation}

When the PSS is perturbed by additive noise of different strengths, the noise effect on figures of merit at different sequence lengths is shown in Fig. 4. The noise performance is examined for different values of $\eta$ ranging between $0 \mathrm{db}$ to $-20 \mathrm{db}$. The noise performance results clearly show that the PSS exhibits high noise robustness at the higher sequence lengths.

\section{Doppler Tolerance}

As explained in Sec. II, when the target has a constant motion, a linear phase shift given by $d \phi=\sigma \pi / N, 0<\sigma \leq 1$ proportional to target velocity will be added on to the received decoded sequence. The performance of poly-semantic sequences in terms of figure of merit without additive noise is shown in the Fig. 5 at different Doppler phase shifts $\left(\varphi_{\mathrm{d}}\right)$ in the interval of $[0.4 \pi / \mathrm{N}, 0.8 \pi / \mathrm{N}]$ per sub-pulse. It is observed from the figure that as Doppler shift increases above $0.5 \pi / \mathrm{N}$, the performance of figure of merit deteriorates.

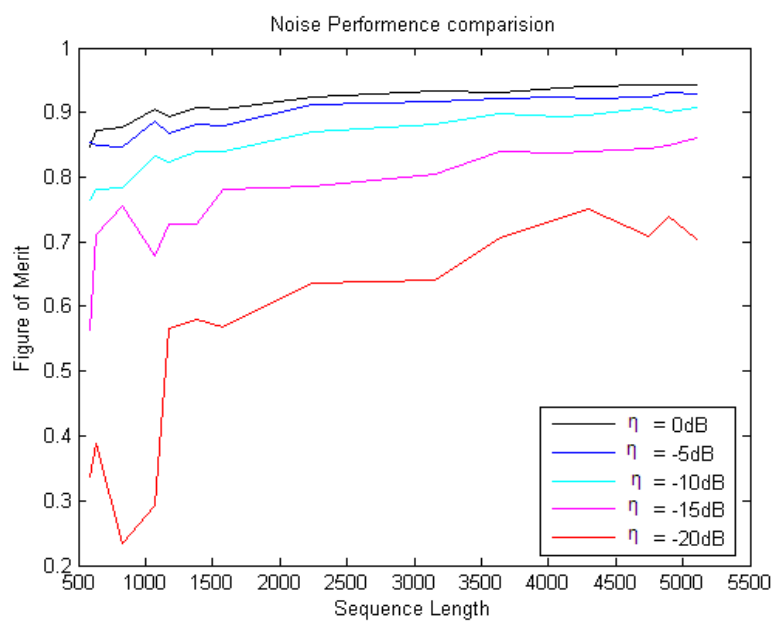

Fig. 4 Noise Performance of Poly-Semantic Sequences

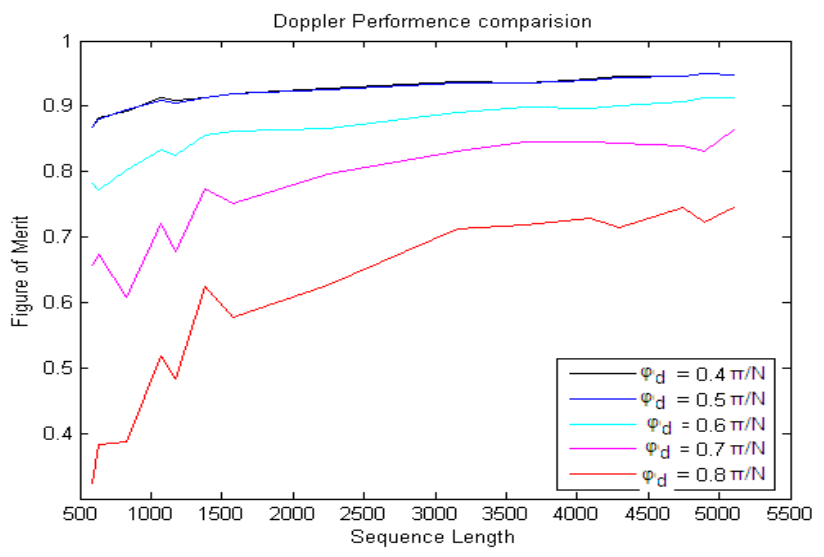

Fig. 5 Doppler Performance of Poly-Semantic Sequences 
When Doppler phase shift increases to $0.8 \pi / \mathrm{N}$, the figure of merit falls below 0.3 (Fig. 5). Thus the information due to target will be masked and it is not possible to identify the target. The proposed sequences have Doppler tolerance up to $0.7 \pi / \mathrm{N}$ with corresponding figure of merit of 0.3 .

\section{Combined Effect of Noise and Doppler Shift}

When the signal encounters the joint effect of additive noise and Doppler shift due to a moving target, the phase shift variation in the received signal becomes non-monotonic function as shown in Fig. 2(c). In such a case some of the sub-pulses (randomly) in the sequences may have phase shift more than $0.5 \pi / \mathrm{N}$. At threshold detection these sub-pulses undergo phase reversal. The performance of figure of merit decreases with the increase of such erroneous bits in the decoded sequence. This results into deterioration in the performance of PSS detection. Fig. 6 gives the figure of merit of PSS sequences at different lengths $N=500$ to 5000 at $\eta=0$ $\mathrm{dB}$ and varying Doppler shift in the interval $[0.4 \pi / \mathrm{N}, 0.8 \pi / \mathrm{N}]$.

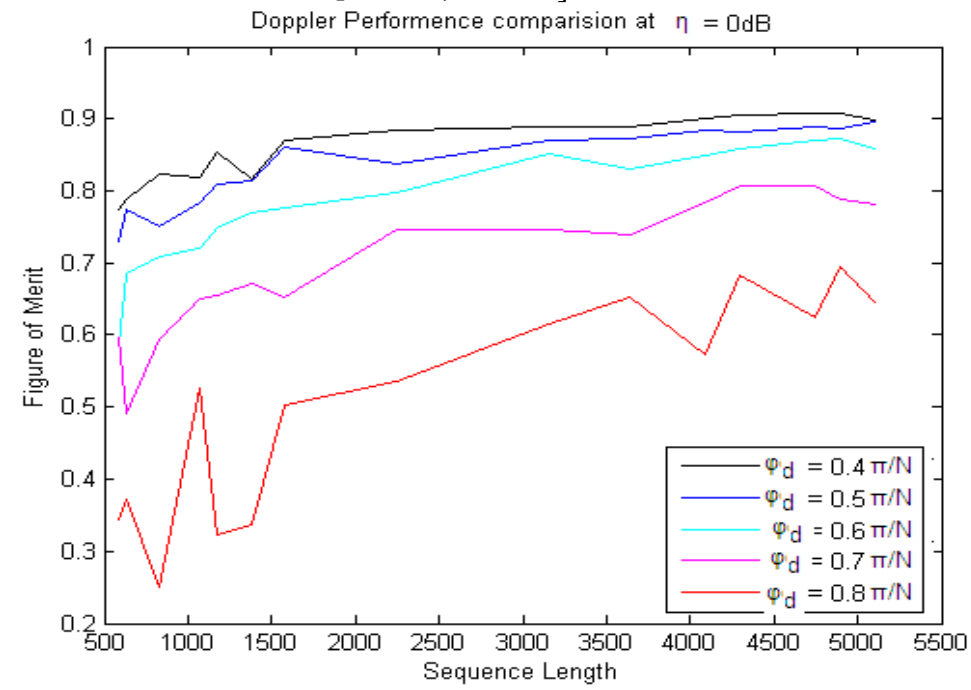

Fig. 6 Noise and Doppler Performance of Poly-Semantic Sequences at Different Doppler Phase Shifts $\left(\Phi_{d}\right)$ and Fixed $\eta$ of $0 \mathrm{~dB}$.

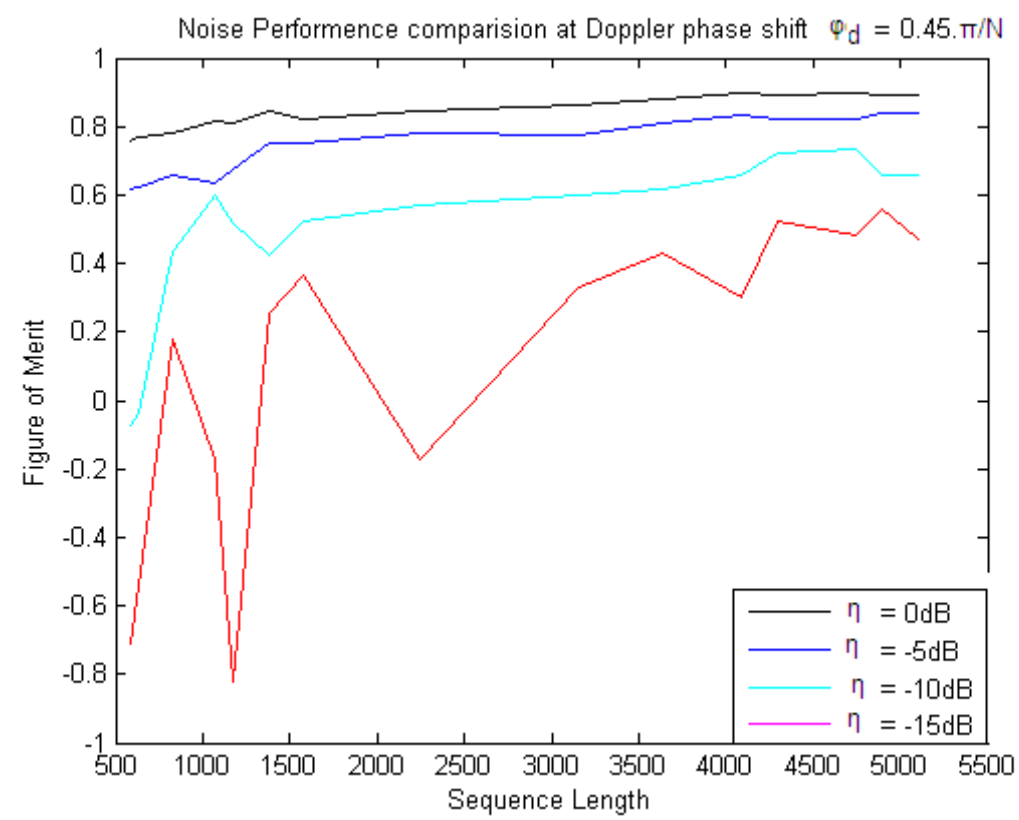

Fig. 7 Noise and Doppler Performance of Poly Semantic Sequences at Different Noise Levels of $\eta$ and Fixed Doppler Phase Shift of $0.45 \pi / \mathrm{N}$. 
Also, Fig. 7 shows at fixed Doppler shift of $0.45 \pi / \mathrm{N}$ and varying $\eta$. It is observed that the sequences exhibits more Doppler tolerance at higher length when compared to lower length since the phase variation per bit is small at higher lengths.

\section{Range Resolution}

Here, we have considered only two point targets, which are moving with same velocity within the resolution volume. The following target model is developed with Doppler shift in noisy environment. This model is simulated using MATLAB functions. Two targets, which are separated from different sub-pulses delay apart (SPDA) with sub-pulse duration ' $\tau$ ' of $50 \mathrm{~ns}$ (range resolution $7.5 \mathrm{~m}$ ) are considered. In principle, the same procedure can be extended to obtain range resolution for multiple targets, which are separated from one SPDA to (N-1) SPDA. When targets are within the sub-pulse range, the resultant echo signal is the addition of all the echo signals from the targets. Depending on the delay between the echo signals, the length of the resultant sequence increases. Fig. 8 is designed as a simulated target model for generating received code $\mathrm{S}_{\mathrm{R}}$, when two targets are at $\mathrm{n}$ - SPDA $(\mathrm{n}=0,1,2, \ldots, \mathrm{N}-1)$ with Doppler shift $\mathrm{f}_{\mathrm{d}}$ in noisy environment. The binary code $S_{b}$ is clocked (2N-1) times into the target model to get the received code $S_{R}$ of fixed length (2N1). Limiter is used to limit the amplitude levels between \pm 1 .

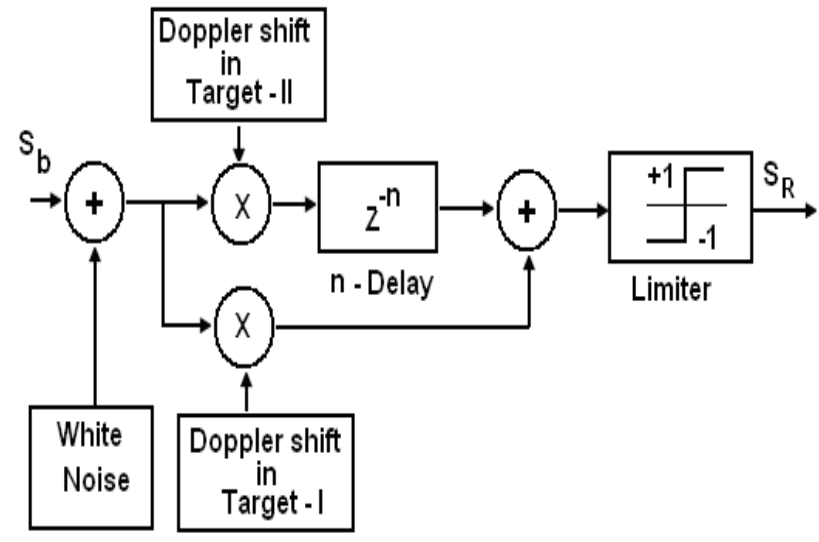

Fig. 8 Target Model When Two Targets are at n-SPDA.

\section{Detection Performance}

Let us consider a $\mathrm{K}_{\mathrm{a}}$-band $30 \mathrm{GHz}$ radar, transmitting a poly-semantic sequence of length $\mathrm{N}=1575$ with pulse interval of $78.75 \mu \mathrm{s}$. The sub-pulse time interval $\tau$ is $50 \mathrm{~ns}$ (signal bandwidth is $20 \mathrm{MHz}$ and range resolution is $7.5 \mathrm{~m}$ ). At the receiver, the resultant waveform is multiply interpreted for coincidence detection. For range resolution ability, consider a target model when a dispersed echo is reflected from two targets located at sub-pulse duration apart (SPDA) of zero to (N-1). Consider two targets moving with the same relative velocity in noisy environment. Fig. 9 shows the poly-semantic sequence of length 1575 when the two targets are separated by 50 SPDA with both Doppler shift $=0.25 \pi / \mathrm{N}$ at different values of (a) SNR ( $\eta)=0 \mathrm{~dB}$ (b) SNR $(\eta)=-5 \mathrm{~dB}(\mathrm{c}) \operatorname{SNR}(\eta)=-10 \mathrm{~dB}$.

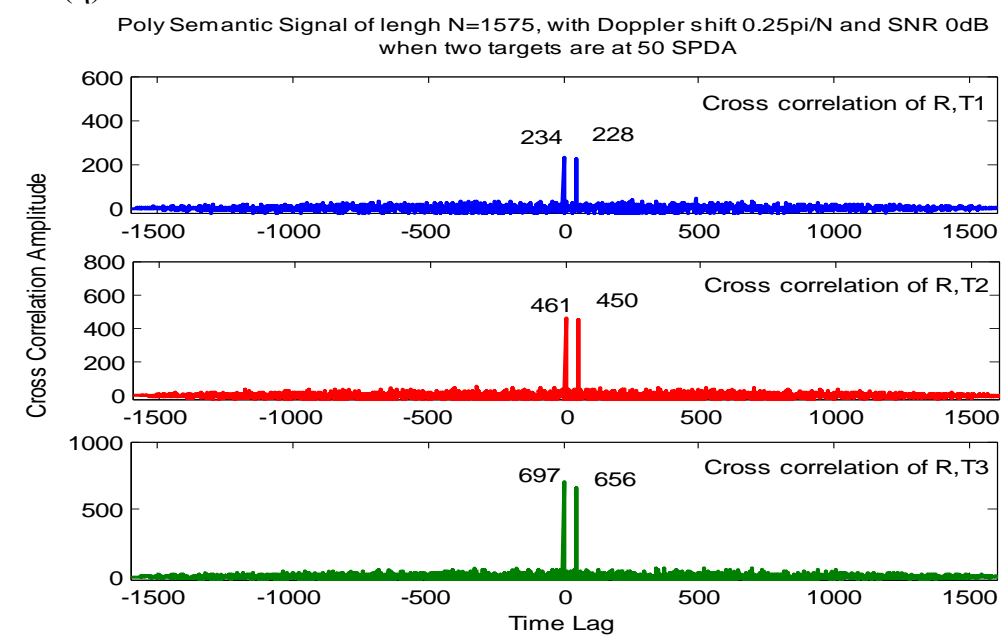

(a) 


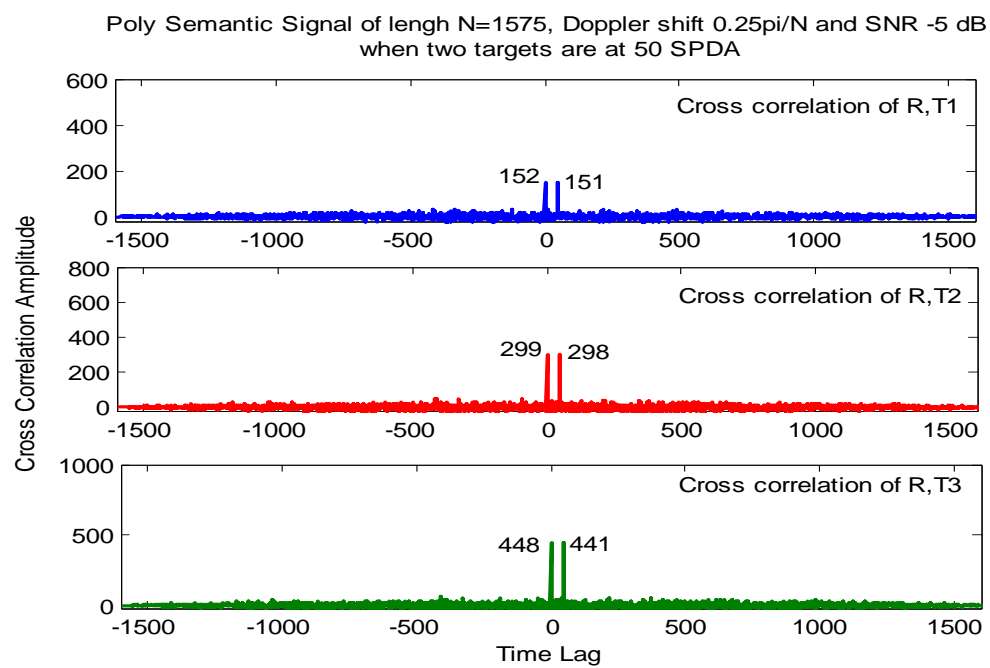

(b)

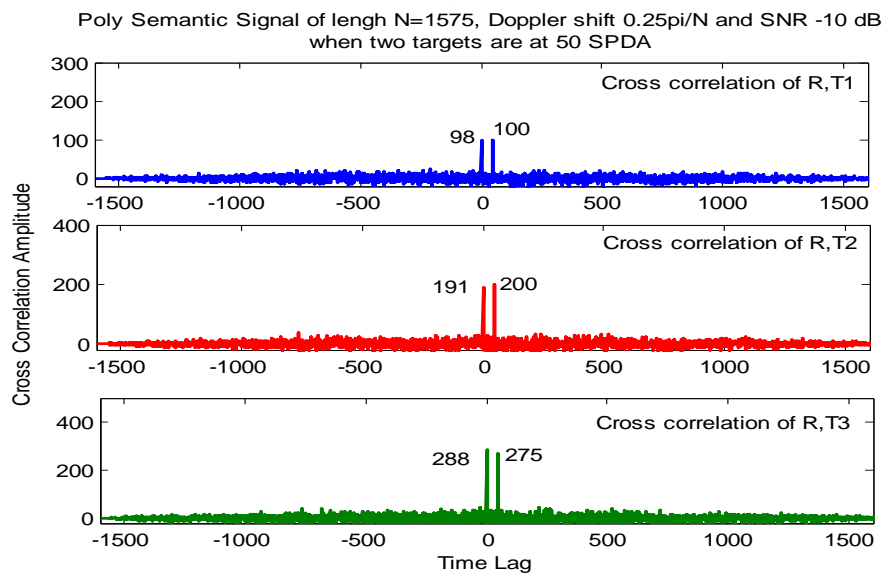

(c)

Fig. 9 Poly-Semantic Sequence of Length 1575 When The Two Targets are Separated by 50 SPDA With Doppler Shift $=0.25 \pi / \mathrm{N}$ at (A) SNR $(\eta)=0 \mathrm{~dB}($ B) SNR $(\eta)=-5 \mathrm{~dB}$ (C) SNR $(\eta)=-10 \mathrm{~dB}$.

\section{CONCLUSION}

In this paper poly-semantic sequences are analyzed for the detection of multiple targets in high density additive noise and Doppler environment for the application of high resolution Doppler radar system. Table I shows that the PSS have higher figure of merit than any other poly alphabetic sequence in noise free environments particularly at larger sequence lengths. These results provide the evidence that the PSS with larger pulse compression ratios can achieve the range side lobe level below $14.78 \mathrm{~dB}$. This is significance improvement over conventional pulse compression sequences [13] and poly-phase alphabetic sequences [6] which provide side lobe level of $13.42 \mathrm{~dB}$ at length $\mathrm{N}>1638$ under noise free environment. This advantage arises because when the binary sequence is designed using 2nd order HBT algorithm, it performs recursive search such that the multiple interpretations of PSS of larger length reinforce each other through matched filtering and coincidence detection. The PSS has significant advantage of noise interference and Doppler tolerance with $\eta$ below $20 \mathrm{~dB}$ at length $\mathrm{N}>4000$. Another important advantage of PSS is that their detection ability is further improved in noise free or noisy environment through coincidence detection scheme. The polysemantic sequences at higher lengths with coincidence detection has noise tolerance of $\eta=-10 \mathrm{~dB}$ with Doppler tolerance of $0.25 \pi / \mathrm{N}$. While compared with poly-phase sequences, a poly-semantic sequence has achieved better noise rejection ability, higher range resolution and superior Doppler tolerance. These examining results lead PSS to be very suitable for the high resolution Doppler radar systems. However, these advantages will be achieved with an additional affordable signal processing at the receiver. 


\section{References}

[1] R. J. Keeler, c. A. Hwang, "pulse compression for weather radar", in proc. Ieee int. Radar conf., 1995, pp. 529-535.

[2] F. B. Dah, c. F. Juang, and c. T. Lin, "a neural fuzzy network approach to radar pulse compression", ieee trans. On geosciences and remote sensing letters., vol. 1, no. 1, 2004, pp. 15-20.

[3] M. J. E. Golay, "the merit factor of long low autocorrelation binary sequences", ieee trans. Inf. Theory it-28: 1982, 543-549.

[4] P. Z. Peebles, j. R., "radar principles", john wiley and sons, inc., 2004

[5] Pasha, n. Sudershan rao, and p. S. Moharir, "poly-alphabetic pulse compression radar signal design", modeling, measurement \& control, journal of amse, vol. 74, no. 4, 2001, pp. 57-64.

[6] Y. Mallikarjuna reddy, i. A. Pasha, and s. Vasthsal, "poly-alphabetic radar signal processor for efficient target detection", international radar symposium i, bangalore, 2007, pp. 30-34.

[7] Pasha, p. S. Moharir, and n. Sudarshan rao, “ bi-alphabetic pulse compression radar signal design”, sadhana-proc. Engg. Sci. Ind. Acad. Sci., vol. 25, 2000, pp. 481-488.

[8] K. Deergha rao and g. Sridhar, "improving performance in pulse radar detection in using neural networks", ieee trans. Aerosp, electron sys, vol. 30, 1995, pp. 1193-1198.

[9] H. K. Kawn and c. K. Lee, "pulse radar detection using a multilayer neural network", proc. Internat. Joint conf. On neural networks,vol. 2, 1989, pp. 75-80.

[10] H. K. Kawn and c. K. Lee, “a neural network approach to pulse radar detection”, ieee trans.aerospace electron. Sys, vol. 29, 1993, pp. 9-21.

[11] Pasha, p. S. Moharir, and v. M. Pandharipande, "poly-semantic binary pulse compression radar sequences", ieee tencon 2003, ieee technical conference on convergent technologies for the asia-pacific, 2003.

[12] P. S. Moharir, k. Rajarajeswari, and k. Venkata rao, "new figures of merit for pulse compression sequences", journal of iete, vol. 38, no. 4, 1992, pp. 209-216.

[13] K. Rajarajeswari. Et al, "new figures of merit for range resolution radar using hamming and euclidean distance concepts", proceedings of the $7^{\text {th }}$ wseas international conference on multimedia systems \& signal processing, hangzhou, china, april 15-17, 2007, pp. 139-145. 